\title{
CARBON MICROSTRUCTURES FOR ELECTROCHEMICAL STUDIES
}

\author{
Robert Kostecki, Xiang Yun Song and Kim Kinoshita \\ Environmental Energy Technologies Division, and \\ Lawrence Berkeley National Laboratory \\ Berkeley, California 94720
}

Thin layers of photoresist were spin coated onto silicon wafers, and then carbonized to form smooth carbon films by heating in nitrogen for 1 hour at temperatures between 600 to $1100^{\circ} \mathrm{C}$. Well-defined carbon microstructures on $\mathrm{Si}$ wafers that are being considered for electrodes in a microbattery concept were obtained by additional processing steps involving patterning and lithography of the photoresist prior to carbonization. The status of the fabrication of carbon microelectrodes obtained by pyrolysis of photoresist, characterization of the carbons by surface-sensitive techniques and electrochemical analysis by cyclic voltammetry of the $\mathrm{I}^{-} / \mathrm{I}_{3}{ }^{-}$redox reaction is described.

Key words: carbon microelectrode, Raman spectroscopy, $\mathrm{I}^{-} / \mathrm{I}_{3}{ }^{-}$redox reaction, photoresist

\section{INTRODUCTION}

Carbon-based materials are available in a variety of physical structures-large solid blocks, highly porous solid particles, fibers, small particles, etc. To produce these carbon structures requires different precursors and manufacturing processes. The precursor may be a gas, liquid, or solid which is heated at temperatures ranging from about $500^{\circ} \mathrm{C}$ to near $3000^{\circ} \mathrm{C}$. The fact that a large number of carbons are available poses a problem for selecting the most appropriate one for use in electrochemical systems. It is through an understanding of the physicochemical properties and electrochemical behavior of carbon that a rational selection is possible.

It is apparent from this discussion that a wide range of carbon materials, with vastly different physicochemical properties are available. Because many carbons are available, a thorough knowledge of their physicochemical and electrochemical properties is necessary to select the most suitable material for use in electrochemical technologies. The traditional electrochemical applications for carbon in solid electrode structures for the chlor-alkali industry (as well as in aluminum refining) are giving way to more diverse applications requiring high-surface-area carbon (i.e., capacitors, fuel cells, metal/air batteries, and high-energy lithium batteries). In each of these applications carbon has the desirable combination of acceptable electrical conductivity, chemical/electrochemical compatibility to the surrounding environment, and availability in the appropriate structure for fabrication into electrodes. In addition, the low cost of carbon relative to other electronic conductors is an important advantage for its widespread use in electrodes, particularly in electrochemical systems that must compete with existing technologies.

Carbon is an integral part of many battery electrodes. In some electrodes, it plays a passive role as a conductive matrix (e.g., positive electrode containing metal oxides), and in others it is an active component as an electrocatalyst (e.g., positive electrode in 
$\mathrm{Zn} /$ air cells) or as the host for electroactive species (e.g., $\mathrm{Li}^{+}$ions in negative electrode for Li-ion batteries). Because carbons are available in a wide variety of morphologies and crystallographic structures, there are many opportunities to demonstrate the viability of carbon in battery applications. One approach that we are exploring is the use of semiconductor-processing techniques that involve photolithography to pattern photoresists and subsequent pyrolysis to form carbon microstructures that function as microelectrodes (1-4). In this paper, we describe the status of the fabrication of carbon microelectrodes obtained by pyrolysis of photoresist and characterization of the carbons by surface-sensitive techniques and electrochemical analysis by cyclic voltammetry. Both carbon films and microelectrode structures were fabricated from pyrolyzed photoresist, and electrochemical results obtained with these carbon materials will be presented.

\section{EXPERIMENTAL}

Both positive (AZ-4330, Hoechst Celanese, Somerville, NJ and OIR-897, Olin Corp., Norwalk, CT)) and negative (XPSU, Microchem. Corp., Newton, MA) photoresists were pyrolyzed to form carbon structures. Thin layers of photoresist were spin coated onto $10-\mathrm{cm}$ diameter silicon wafers, using either manual or automated control. After curing and baking, the photoresists were carbonized to form smooth carbon films by heating in nitrogen for 1 hour at temperatures between 600 to $1100^{\circ} \mathrm{C}$. A similar procedure was used to produce well-defined carbon microstructures on Si wafers by additional processing steps involving patterning and lithography. Carbon microstructures were fabricated to form interdigitated electrodes (IDEs) with the following dimensions: length of the digits of $\sim 500 \mu \mathrm{m}$, width of the digits of $\sim 50 \mu \mathrm{m}$, and distance between digits of $\sim 10 \mu \mathrm{m}$. The carbon IDEs are thin carbon films $(<10 \mu \mathrm{m}$ thickness) that are patterned on a Si wafer and are essentially two-dimensional structures. As an extension of our efforts to fabricate carbon microelectrodes, additional processing steps were performed to fabricate three-dimensional carbon microstructures ("carbon post"). Carbon structures of cylindrical geometry ( $\sim 0 \mu \mathrm{m}$ diameter and $\sim 6 \mu \mathrm{m}$ height) were fabricated on the interdigitated carbon fingers by the procedure described elsewhere (3). Except for the final heat-treatment steps, the procedures involving patterning and photolithography were carried out in the Microfabrication Facility in the Electronics Research Laboratory on the campus of the University of California at Berkeley.

Raman spectroscopy (RS) measurements were carried out at room temperature in ambient atmosphere using an argon-ion laser (Coherent Inc. Model Innova 70) tuned to $514.5 \mathrm{~nm}$. An atomic force microscope (Molecular Imaging) with an electronic controller (Park Scientific Instruments) was used to study the surface topography of the carbon surface. A surface profile analyzer (New View System 200, Zygo Corp., Middlefield, CT), which combines optical microscopy and interferometry was used to obtain images of the microstructure and 3-D surface topography.

Redox couples, such as $\mathrm{I}_{3}-/ \mathrm{I}^{-}$and $\mathrm{Fe}(\mathrm{CN})_{6}{ }^{4-} / \mathrm{Fe}(\mathrm{CN})_{6}{ }^{3-}$, were used to investigate the electrochemical response of the carbon materials. A more detailed description of the electrochemical cell and experimental conditions is presented by Kostecki et al. $(2,4)$.

\section{RESULTS AND DISCUSSION}


Fabrication of Microelectrode Arrays. A schematic representation of the processing steps used to fabricate the microelectrode arrays is illustrated in Figure 1. Starting from the top of the figure, the steps involve:

Fig. 1. Schematic representation of the processing steps to fabricate threedimensional carbon microstructures. Left - top view. Right - side view.

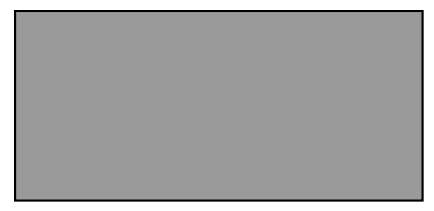

CVD of Si 3N4 layer on Si wafer
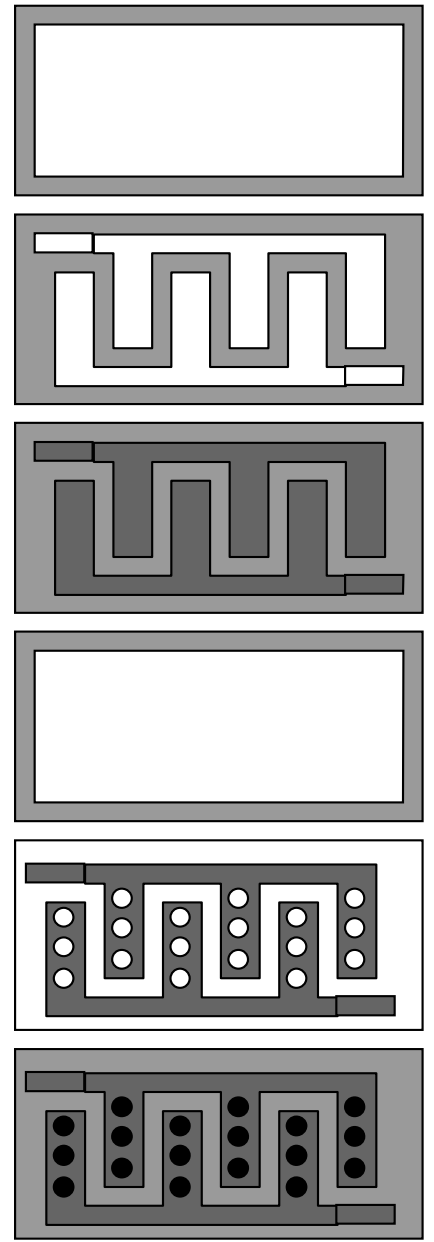

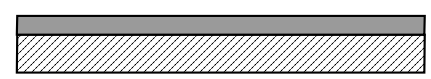

Spin coat photoresist on Si3N4/Si wafer

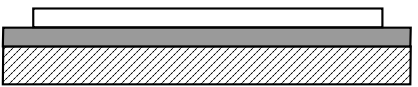

Pattern current collector by photolithography

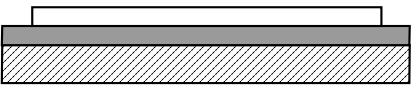

Heat treat to form carbon current collector

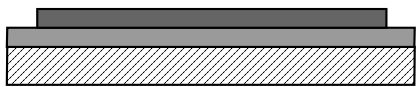

Spin coat second layer photoresist

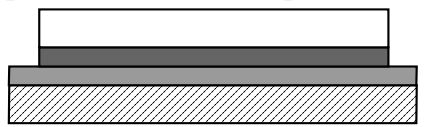

Pattern post structure by photolithography

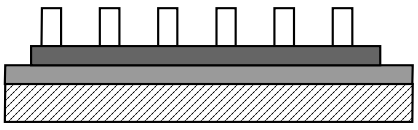

Heat treat to form carbon post structure

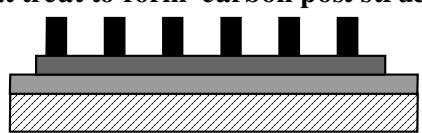

Step 1. CVD silicon nitride layer ( $100 \mathrm{~nm}$ thick) on silicon water.

Step 2. Spin coat photoresist on silicon nitride layer.

Step 3. Pattern photoresist by photolithography to form interdigitated arrays.

Step 4. Develop and process photoresist. (not shown in Fig. 1)

Step 5. Heat treat wafer to carbonize photoresist to form interdigitated current collector arrays.

Step 6. Spin coat second layer of photoresist. 
Step 7. Pattern photoresist by photolithography to form posts.

Step 8. Develop and process photoresist. (not shown in Fig. 1)

Step 9. Heat treat wafer to carbonize photoresist to form carbon posts.

In the figure, white represents the photoresist before heat treatment. The carbon obtained by heat treatment is depicted by dark grey (Step 5) and black (Step 9). Alternatively, Step 5 can be eliminated, and only one carbonization process (Step 9) can be used to form both the carbon fingers and posts. Both spectroscopic/microscopic examination and electrochemical studies were conducted on carbon obtained following Steps 5 and 9. These results are presented in the following sections.

Characterization of Carbon Films and Microstructure. An example of a computergenerated cross-section profile of a three-dimensional carbon microstructure obtained by optical microscopy is shown in Fig. 2. The microstructure was obtained by heat treatment of the photoresist at $1000^{\circ} \mathrm{C}$ following the steps outlined in Fig. 1. Optical microscopy indicated that the interdigitated carbon structures have an irregular profile, with the cross section usually having a cone shape. In this example, the carbon posts are roughly $6-\mu \mathrm{m}$ tall and about $16-\mu \mathrm{m}$ diameter. The peak-to-peak separation is approximately $55 \mu \mathrm{m}$. The current collector is also evident at the base of the carbon posts in Fig. 2; they are about $28-\mu \mathrm{m}$ wide. The threedimensional carbon structures were more difficult to fabricate reproducibly than the interdigitated carbon fingers. In some instances, the carbon posts resembled pyramid structures and/or were poorly located. In other words, misalignment resulted in part of the posts located between the carbon fingers. However, we were able to fabricate acceptable threedimensional carbon microstructures that were considered for electrochemical studies.

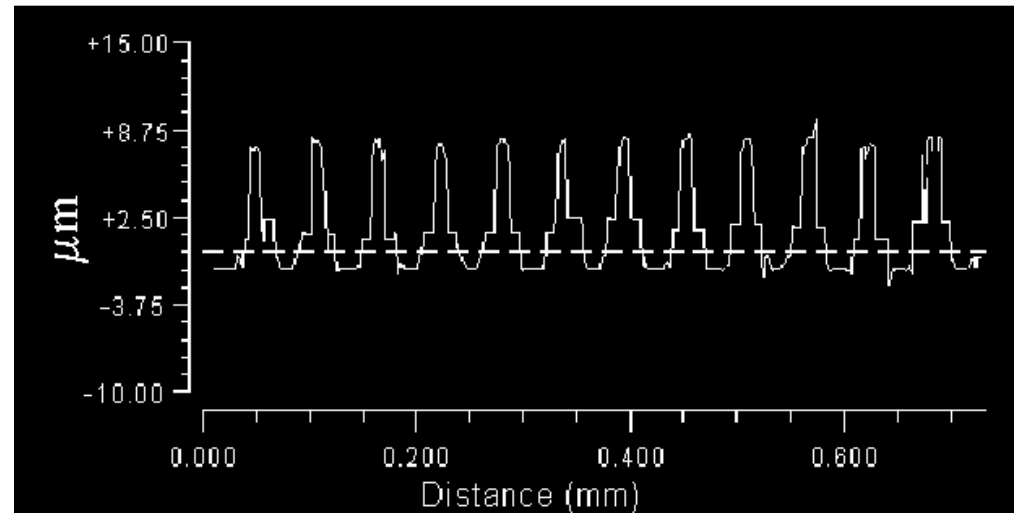

Fig. 2. Computer-generated display of cross section of threedimensional carbon microstructure using optical microscope

Atomic force microscopy (AFM) studies showed that carbon films produced following Steps 1-5 have relatively smooth surfaces with an estimated roughness of about $3 \mathrm{~nm}(1-4)$. The AFM images of the carbon films reveal a compact surface structure. No AFM studies were conducted on carbon structures obtained after Step 9. Instead we relied on the surface profile analyzer to observe the carbon structure.

An optical microscope image of a section of the carbon posts on interdigitated carbon fingers is shown in Fig. 3. The dark spheres are the carbon posts, and the carbon fingers are the horizontal dark regions connected to the posts. A micro-probe Raman spectrometer was used to investigate the carbon spectra associated with the carbon 
current collector (i.e., carbon obtained following Steps 5 and 9) and the carbon post. The Raman spectra that were obtained on the carbon fingers and carbon posts are presented in Fig. 3. These Raman spectra of the carbon materials represent the general behavior of vibrational spectra obtained from highly disordered carbonaceous materials. The spectrum shows a strong and band at $1355 \mathrm{~cm}^{-1}$ with a broad $\mathrm{E}_{2 \mathrm{~g}}$ graphite peak shifted toward higher frequencies $\left(\sim 1600 \mathrm{~cm}^{-1}\right)$. The intensity of the Raman peaks varied with the heat-treatment temperature that was used to pyrolyze the photoresist. A detailed discussion of the Raman spectroscopic studies of carbon films is presented elsewhere (5).

Electrochemical Studies of Carbon

B

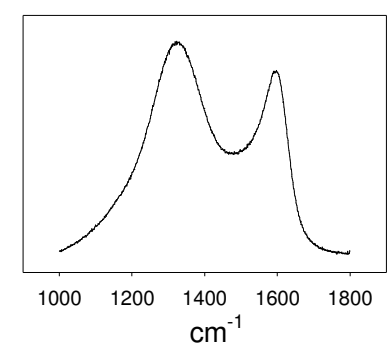

A

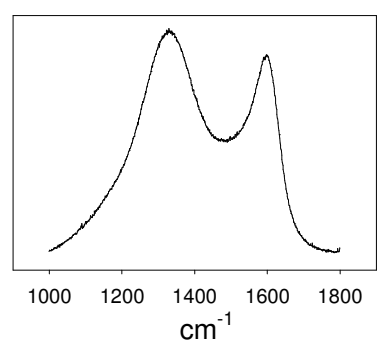

Fig. 3. Optical microscope image and Raman spectra obtained on the carbon finger (A) and carbon post (B)

Films and Microstructures. The

kinetics for the $\mathrm{Fe}(\mathrm{CN})_{6}{ }^{4-} / \mathrm{Fe}(\mathrm{CN})_{6}{ }^{3-}$ redox couple on carbon films are strongly dependent on the temperature at which the photoresist is pyrolyzed (1). The results clearly show that carbon films obtained by pyrolysis of a photoresist at temperatures $700^{\circ} \mathrm{C}$ have glassy-carbon-like behavior with good electrochemical reversibility. Carbon films obtained by pyrolysis at $1000^{\circ} \mathrm{C}$ show even better electrocatalytic properties than glassy carbon. The electrochemical studies on carbon films was extended to measurements of carbon interdigitated electrodes (IDE) obtained by the above procedure to Step 5.

The $\mathrm{I}^{-} / \mathrm{I}_{3}{ }^{-}$redox reaction was used to investigate the electrochemical response of the carbon IDEs. The redox behavior of the carbon IDEs observed by cyclic voltammetry indicated that these microelectrodes are viability alternatives for a ring-disc electrode. This is illustrated in Fig. 4 which shows the current at one of the carbon IDEs (collector electrode potentiostatically controlled at $-0.1 \mathrm{~V}$ ) while a linear potential sweep $(1 \mathrm{mV} / \mathrm{s})$ between 0.0 and $0.8 \mathrm{~V}$ was applied to the other carbon IDE (generator electrode). The interdigitated array was arranged in the single-cell geometry (see Fig. 2 in ref. 2). In this experiment, $\mathrm{I}_{3}{ }^{-}$is produced at the generator electrode and detected at

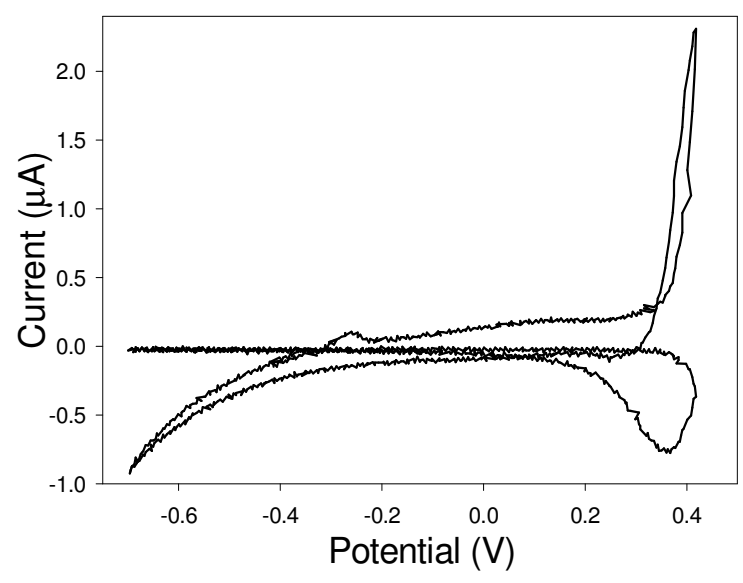

Fig. 4. Current response of collector electrode $(-0.1 \mathrm{~V})$ to $\mathrm{I}_{3}{ }^{-}$produced at the generator electrode during potential scan at $1 \mathrm{mV} / \mathrm{s}$ in $0.1 \mathrm{M} \mathrm{KI}$. 
the collector electrode which is set at a potential where $\mathrm{I}_{3}{ }^{-}$is reduced to $\mathrm{I}^{-}$ions. The collection efficiency determined from the slope of the collector current and generator current is $\sim 0.91$, which is substantially higher than the collection coefficients (i.e., varies from 0.2 to 0.4 ) typically observed with rotating ring-disc electrodes $(6,7)$. The electrochemical response of the IDEs is dependent on the geometric arrangement of the interdigitated structure (4).

The electrochemical kinetics involving the $\mathrm{I}^{-} / \mathrm{I}_{3}{ }^{-}$redox reaction was studied with the microelectrode arrays consisting of the three-dimensional carbon microstructure. An unexpected problem was encountered that precluded meaningful electrochemical measurements of the $\mathrm{I}^{-} / \mathrm{I}_{3}{ }^{-}$redox reaction using generator and collector electrodes, as described above. An electrical short-circuit was found in many of the arrays of threedimensional carbon microstructure. The origin of this problem has not been resolved.

\section{CONCLUSIONS}

Semiconductor processing steps involving spin-coating photoresist, photolithography and carbonization to form $\mu \mathrm{m}$-size nanostructures for carbon electrodes were successfully accomplished. Photoresist layers of about $10-\mu \mathrm{m}$ thickness were spincoated on silicon wafers and carbonized at temperatures from $500-1100^{\circ} \mathrm{C}$ in an inert atmosphere to form three-dimensional carbon microstructures. Electrochemical studies were conducted to characterize the carbon electrodes by redox reactions involving the ferri/ferrocyanide and iodine/iodide redox couples. Cyclic voltammetry clearly shows that the electrochemical behavior of the carbon depends strongly on the carbonization temperature - improved performance is obtained with samples produced at higher temperature. The close arrangement of the carbon fingers yields an electrochemical response that is similar to that of a rotating ring-disc electrode for detecting ionic species in solution. Electrochemical studies with three-dimensional carbon microstructures were not successful because of electrical short circuits between the two electrodes in the array.

\section{ACKNOWLEDGMENT}

This work was supported by the Director, Office of Energy Research, Office of Basic Energy Sciences, Chemical Sciences Division of the U.S. Department of Energy under Contract No. DE-AC03-76SF00098.

\section{REFERENCES}

1. J. Kim, X. Song, K. Kinoshita, M. Madou and R. White, J. Electrochem. Soc., 145, 2314 (1998).

2. R. Kostecki, X. Song and K. Kinoshita, Electrochem. and Solid-State Lett., 2, 461 (1999).

3. K. Kinoshita, X. Song, J. Kim and M. Inaba, J. Power Sources, 81-82, 170 (1999).

4. R. Kostecki, X. Song and K. Kinoshita, J. Electrochem. Soc., 147, 1879 (2000).

5. R. Kostecki, B. Schnyder, D. Alliata, X. Song, K. Kinoshita and R. Kötz, submitted to Thin Solid Films (2000). 
6. Ring-Disk Electrode, W.J. Albery and M.L.Hitchman, Oxford University Press, Oxford, UK (1971).

7. The Rotating Disc Electrode, V.Yu. Pleskov and Yu.V. Filinowskii, Consultants Bureau, New York, NY (1976). 